\title{
Head CT in Patient with Metabolic Acidosis
}

\author{
Kavita M. Babu, $M D^{a}$, C. D. Rosenbaum, $M D^{b}$, Edward W. Boyer, $M D, P h D^{b}$
}

aBrown University, Program in Medical Toxicology/Department of Emergency Medicine, Providence, RI

bDivision of Medical Toxicology, Department of Emergency Medicine. University of Massachusetts, Worcester, MA

\section{Answer from page 257: Methanol.}

\begin{abstract}
An unresponsive 30-year-old female with a history of anxiety and chronic alcohol abuse presented to an emergency department with altered mental status and a severe metabolic acidosis. The patient was intubated for airway protection, and she empirically received folic acid, bicarbonate, and 5\% ethanol continuous infusion for suspected ingestion of toxic alcohol. Following transfer to our institution, the patient was minimally responsive to noxious stimuli. She received fomepizole at dosing corrected for hemodialysis (HD), and bicarbonate via multiple boluses and continuous infusion. The ethanol drip was stopped. The nephrology service had been alerted to this patient's arrival and condition; hemodialysis via a standard heparinized circuit was initiated immediately after her arrival, which produced a marked improvement in the patient's acid-base status. Her serum methanol concentration subsequently returned at $>200 \mathrm{mg} / \mathrm{dL}$.

After 12 hours and 2 sessions of hemodialysis, the patient remained unresponsive despite minimal sedation. Anisocoria was noted on exam. Computed tomography of the brain demonstrated a large hematoma in the left basal ganglia that extended into the left frontal and parietal white matter accompanied by intraventricular extension, midline shift, loss of grey-white differentiation throughout, suggesting tonsillar herniation (Figure 1). Forty-eight hours after presentation, radionuclide imaging of the brain revealed no intracranial blood flow; heart, lungs, liver, kidneys, and pancreas were subsequently harvested for transplantation.
\end{abstract}

\section{DISCUSSION}

Putaminal necrosis, with or without hemorrhage, has been described as a characteristic complication of methanol toxicity. This susceptibility of the putamen has been attributed to high metabolic demands and poor venous drainage [1]. The precise mechanism of putaminal injury is unknown; direct effects of methanol, formic acid or other methanol metabolites, ischemic injury and acidosis have all been postulated [2,3]. Hemodialysis of methanol-intoxicated patients against a heparinized dialysis circuit has been associated with several cases of intracranial hemorrhage in the setting of methanol toxicity. However, causality has not been established, and hemorrhage may reflect severity of poisoning rather than heparin effect. At least one patient with severe methanol toxicity demonstrated intracranial hemorrhage prior to onset of hemodialysis [4]. The risks are minimal for short courses of hemodialysis without heparin, and empiric dialysis without heparin in the setting of severe methanol intoxication may warrant consideration in patients without imaging as well as the setting of known intracranial hemorrhage [2]. Patients with catastrophic intracranial hemorrhage secondary to methanol can still be considered for organ donation. Hemodialysis and fomepizole therapy

Keywords: Methanol, dialysis, heparin, hemorrhage

Note: There was no outside funding of any kind used for this study.

Corresponding Author: Kavita M. Babu, MD, Brown University, Program in Medical Toxicology/Department of Emergency Medicine, 593 Eddy St., Providence, RI 02903 Email: kavitambabu@gmail.com 
should be continued until complete methanol elimination is achieved in viable donors with appropriate consent.

The authors have no potential financial conflicts of interest to report.

\section{REFERENCES}

1. Blanco M, Casado R, Vazquez F, Pumar JM. CT and MR imaging findings in methanol intoxication. AJNR Am J

Neuroradiol 2006 Feb;27(2):452-544.
2. Hernandez MA, Holanda MS, Tejerina EE, Gonzalez C, Lopez M, Hernandez JL. Methanol poisoning and heparin: a dangerous couple? Am J Emerg Med 2004;22(7):620-621.

3. Phang PT, Passerini L, Mielke B, Berendt K, King EG. Brain hemorrhage associated with methanol poisoning. Crit Care Med 1988;16(2): 137-140.

4. Patankar T, Bichile L, Karnad D, Prasad S, Rathod K. Methanol poisoning: brain computed tomography scan findings in four patients. Australas Radiol 1999 Nov;43(4): 526-528. 